\title{
Wireless Battery Charger for EV with Circular or Planar Coils: Comparison
}

\author{
L.Romba $^{1}$, E. Baikova ${ }^{1,2}$, C. Borges ${ }^{1}$, S.S. Valtchev ${ }^{1}$, R. Melicio ${ }^{3,4}$ \\ ${ }^{1}$ UNINOVA-CTS and FCT, Universidade Nova de Lisboa \\ ${ }^{2}$ EST Setúbal, Instituto Politécnico de Setúbal, Setúbal \\ ${ }^{3} \mathrm{ICT}$, Universidade de Évora, Escola de Ciências e Tecnologia, Departamento de Física \\ ${ }^{4}$ IDMEC, Instituto Superior Técnico, Universidade de Lisboa (ruimelicio@gmail.com)
}

\begin{abstract}
This paper presents the experimental results obtained in the wireless energy transfer (WET) system prototype based on coils: circular or planar. With these experimental results we can choose the tuning settings to improve the efficiency of power transmission of the WET systems. In WET for electric vehicle batteries charging, the coil shape and the range between the coils are the most important issues of those systems.
\end{abstract}

Keywords: Wireless Power Transfer, magnetic coupling, planar coil, circular coil, V2G, V2H, V2B.

\section{Introduction}

The WET systems are associated with innovative technologies to create new possibilities: to charge batteries of different devices, to eliminate the cables, the plugs and sockets, to increase the reliability, to guarantee a maintenance-free operation of the critical systems. The WET is especially used in biomedical implants, electric vehicles, and robotic systems [1]. The WET technologies are classified in two categories: non-radiative and radiative. The non-radiative category is used at short and mid-range distances. The small devices, like cell phone, require a small amount of power to charge their batteries and their charging systems operate at a short distance, a few millimeters, between the transmitter and receiver. The charging system for electric vehicles is an example of mid-range distance. Both examples are defined as near field or non-radiative technologies. The power in this case, is transferred by magnetic field using inductive coupling between coils. The long-range or far-field energy transfer is a radiative technology. The power is transferred by electromagnetic waves like microwaves or laser beams. An example of that far-field technology is the solar power satellite [2].

The near field is usually considered to the one at a distance up to $(1 / 2 \pi) \lambda$, that is $0.159 \lambda$ ( $\lambda$ is the wavelength of the frequency generated by the source) [3-5]. The essence of the mid-range WET is the inductive coupling through a strong magnetic coupling (SMC). The inductive coupling process allows for an electric energy transfer using only the magnetic field. Originally, the strong magnetic coupling technology was proposed in 2007 by Marin Soljacic. This pioneer technology has broken the 
traditional WET model for inductive power transfer (IPT) process for which the efficiency strictly depends on the coupling coefficient $k$ between the coils. The energy transmission distances were extended from a few millimetres to distances of some meters. The possibility to stretch the distance represented a significant advance in WET systems [4-6]. The strong magnetic coupling diagram is shown in Fig. 1.

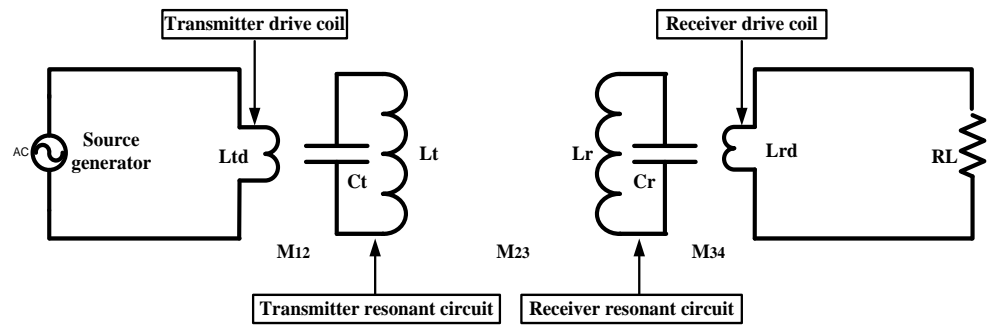

Fig. 1. Strong magnetic coupling diagram.

In Fig. 1 the electric structure used in this technology contains the transmitter drive coil and the transmitter resonant circuit $L_{t}, C_{t}$ at the transmitter side, a receiver resonator $L_{r}, C_{r}$ and a load coil (receiver drive coil) at the receiver side. The utilization of two excitation coils involves two additional mutual coupling coefficients $M_{12}, M_{34}$. The mutual coupling between the two resonators is referenced by $M_{23}$. Those two extra coefficients introduce extra freedom in spreading the transfer distance. However, the overall transfer efficiency will not exceed $50 \%$ because of the independence matching requirement [7]. It is important to differentiate two basic concepts, namely: 1) the maximum power transfer principle; and 2) the maximum energy efficiency principle. The impedance matching method adopted in many WET projects is based on the maximum power transfer theorem. The maximum power transfer principle requires impedance matching between the source and the load. The maximum power theorem applies to a situation in which the source impedance is fixed. When the maximum power transfer occurs at impedance matching, in WET systems, the maximum energy efficiency under the maximum power transfer approach never exceed 50\% [8].

A coil size problem has been observed in systems of simultaneous charge of batteries in portable devices, e.g. mobile phones, laptops, and electric vehicles. The use of planar coils has minimized the negative impacts of the use of circular coils for certain applications. In this paper, the magnetic field created by the two types of circular and planar coils is compared. The tuning control process of the transmitter and the receiver resonant circuits, with these two types of coils, is also investigated.

\section{Technological Innovation for Resilient Systems}

The last decades have been challenging due to the constant uncertainties motivated by the unpredictability of catastrophic climatic events, terrorist attacks, to name a few. Those events have caused social unrest in which almost everything is uncertain [9]. The recent natural disasters have alerted the societies to the interdependence and functionality of critical structures. Failures in interdependent structures can cause 
cascade effects on critical structures. The critical infrastructures are highly interconnected and mutually dependent in complex ways. What happens to one infrastructure can directly and indirectly affect other infrastructures, with impact in large geographic regions. The most important challenges are to understand, identify and analyze the interdependencies of critical national infrastructures. The production, transportation and distribution of electric power is an example of a basic infrastructure that affects our daily lives. Any disruption in this area has implications for the entire social system as a fundamental service for the security and social wellbeing of any nation $[10,12]$. Nowadays the concept of sustainability has been replaced by the concept of resilience. The National Infrastructure Advisory Council in the Critical infrastructure resilience final report a recommendations defines critical infrastructure resilience as: “...the ability to reduce the magnitude and/or duration of disruptive events. The effectiveness of a resilient infrastructure or enterprise depends on its ability to anticipate, absorb, adapt to, and/or rapidly recover from a potentially disruptive event."

The energy industry is changing: renewables and decentralized energy sources, the need for continuous grid optimization to name only a few. New business models have to be developed and new players are emerging. The future smart grids ambient and cyber-physical systems have to have a layered of cyber infrastructures accessing resilient power application that are able to give security and reliability, having the ability to act in order to maintain and correct infrastructure components without affecting the service [11]. A new approach in terms of decentralized energy sources can be achieved through purely electric vehicles and plug-in hybrid vehicles. Depending on the model, each electric vehicle can stored approximately between $5 \mathrm{kWh}-40 \mathrm{kWh}$ of energy. This energy can be used not only to travel but purposes but also for other appliances like providing energy for homes (V2H), or buildings (V2B) and even for supply ancillary services to distribution grid operators, through concept (V2G). These vehicles can charge the batteries overnight, and provide the stored energy during the day as required, to the grid, for a building or for a home from a supply point in a parking lot. The importance of this new concept is most evident in the case of a disruption of the electrical power system, being able to support essential services for some time [13].

\section{Modeling}

\subsection{Circular Coil}

The mutual inductance of a pair of coils can be determined experimentally by two measurements of self-inductance, in one of which the current flows in the same direction in the two coils and in the other it flows in opposite directions. If $L$ and $L^{\prime}$ are the two values of self-inductance determined experimentally, $L_{1}$ and $L_{2}$ the selfinductances of the two coils separately, and $M$ their mutual inductance [14] is given by:

$$
M=\frac{L-L^{\prime}}{4}
$$


The sum method for determining the inductance of a solenoid assumes that the solenoid is composed of $n$ equal circular coaxial circular rings spaced uniformly at a distance corresponding to the pitch $g$ of the winding. Thus, the coaxial length of the coil is $n g$. The current is assumed to get from one ring to the next by means of connections of negligible inductance; that is, the helicity of the actual winding is neglected, the current being assumed to flow in planes perpendicular to the axis of the coil. The inductance of the coil [15] is then given by:

$$
L=n L_{1}+2 \sum_{1}^{n-1} M_{p}
$$

Where $L_{l}$ is the inductance of a turn, and $M_{p}$ is the mutual inductance of two turns separated by a distance $p g$. Using the accurate formula for the inductance of a circular ring of a mean radius $a$ having a cross section of radius $\rho$ [15] is given by:

$$
n L_{1}=4 \pi n a\left[\left(1-\frac{\rho^{2}}{8 a^{2}}\right) \log _{e} \frac{8 a}{\rho}+\frac{\rho^{2}}{24 a^{2}}-\frac{7}{4}\right]
$$

The mutual inductance $M_{p}$ is obtained by the Maxwell series formula for the mutual inductance of coaxial circular filaments near together [15] is given by:

$$
M_{p}=4 \pi a\left[\left(1+\frac{3}{16} \frac{p^{2} g_{2}}{a^{2}}+\cdots\right) \log _{e} \frac{8 a}{p g}-\left(2+\frac{p^{2} g^{2}}{16 a^{2}}+\cdots\right)\right]
$$

\subsection{Planar Coil}

The expressions for $L, R, C, Q$ and $k$ of flat spiral or planar coils are solved in terms of the outer diameter $D_{o}$, number of turns $N$, spacing between each turn $p$ and wire diameter $W$ of the coil. Although $D_{o}, N, p$ and $W$ fully define a flat, spiral coil, all geometric parameters are shown for completeness shown in Fig. 2. The inner diameter $D_{i}$, total wire length $l$, winding radius $a$, and radial depth of the winding $c$ are defined in (5) to (8) [16]. All units of length are in meters.

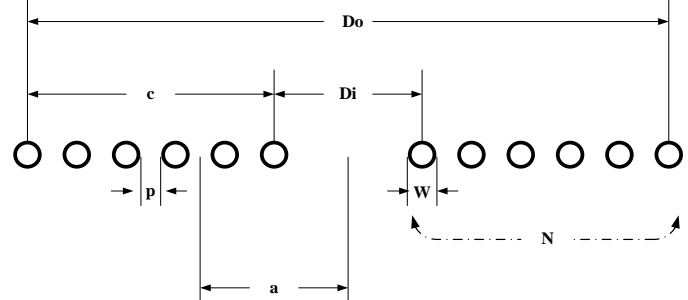

Fig. 2. Cross-sectional view of planar coil [16].

$$
\begin{gathered}
D_{i}=D_{o}-2 N(W+p) \\
\ell=\frac{1}{2} N \pi\left(D_{o}+D_{i}\right) \\
a=\frac{1}{4}\left(D_{o}+D_{i}\right) \\
c=\frac{1}{2}\left(D_{o}-D_{i}\right)
\end{gathered}
$$


The self-inductance for planar coils is given by:

$$
L(H)=\frac{N^{2}\left[D_{o}-N(W-p)\right]^{2}}{16 D_{o}+28 N(W+p)} \times \frac{39,37}{10^{6}}
$$

Where $L$ is derived from a modification of Wheeler's formula for a single-layer helical coil, while accounting for the conversion from inches to meters $39.37 \mathrm{in} / \mathrm{m}$ and $\mu H$ to $H\left(10^{-6}\right)$. In (9) is valid for most geometries except: 1$)$ when the coil has very few turns, 2) when the pitch is very large relative to the wire diameter $(p>>W)$, and 3$)$ when $p>>W$ and $c / a<0.3[16]$.

\section{Case study}

The WET at mid-range distances is carried through the air and based on the magnetic field. Therefore, the study of the magnetic field created by different types of coils is very important $[17,18]$. In this case study the comparison of the magnetic field originated by a planar coil and by a circular coil is made. In addition, a tuning control system is presented based on the magnetic core reactor, which allows tuning of these coils in resonant circuits.

\subsection{Circular Coil}

The circular coil connected in series with MCR and the capacitance (resonant circuit) used in the tests is shown in Fig. 3. This coil is connected in series with the magnetic core reactor (MCR) and the fixe capacitance $C_{t}=0.23 \mu F$ (resonant circuit). The coil is assembled by 35 turns with normal copper cable with a section of $1.5 \mathrm{~mm}^{2}$. The outer diameter is $240 \mathrm{~mm}$. The average thickness of the coil is $35 \mathrm{~mm}$. The cable length used to assembly this coil is $29 \mathrm{~m}$.

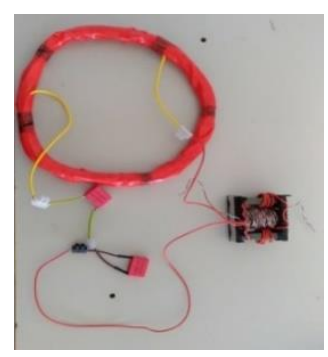

Fig. 3. Circular coil connected in series with MCR and the capacitance (resonant circuit).

The MCR tuning capacity was tested to the frequency range between $15 \mathrm{kHz}$ and $30 \mathrm{kHz}$. The different tuning points when the MCR control DC current ranges from $0.0 \mathrm{~A}$ up to $3.0 \mathrm{~A}$ is shown in Fig. 4 for the above two frequencies. The frequency adjustment range is between the two points of maximum impedance. 


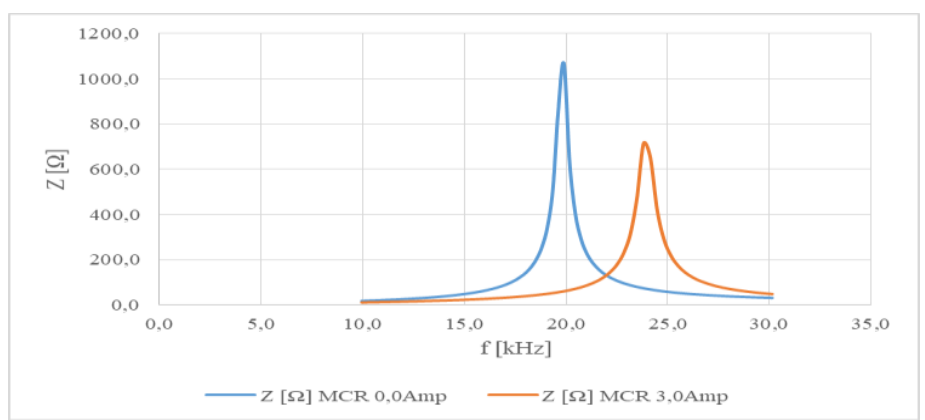

Fig. 4. Tuning points by varying the MCR DC control current for circular coil.

The magnetic field created by this coil (alone) was simulated when it is driven by a current of $15 \mathrm{~A}$ with the frequency of $17 \mathrm{kHz}$. The simulation results for circular coil: magnetic field distribution is shown in Fig. 5.

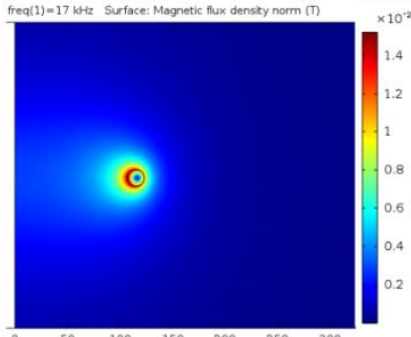

Fig. 5. Circular coil: magnetic field created for the frequency of $17 \mathrm{kHz}$.

\subsection{Planar Coil}

The planar coil connected in series with MCR and the capacitance (resonant circuit) is shown in Fig. 6. This coil is connected in series with the MCR and the fixe capacitance $C_{t}=0.3 \mu \mathrm{F}$. The coil is formed by 35 turns of normal copper cable with a section of $1.5 \mathrm{~mm}^{2}$. The outer diameter is $240 \mathrm{~mm}$ and the inner diameter is $50 \mathrm{~mm}$. The average pitch coil is $2 \mathrm{~mm}$. The cable length used to assembly this coil is $18 \mathrm{~m}$.

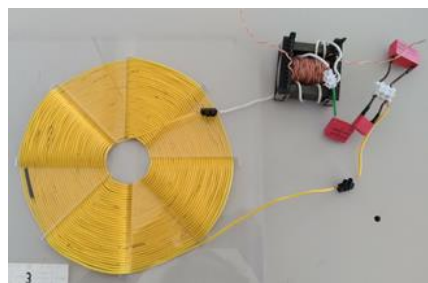

Fig. 6. Planar coil connected in series with MCR and the capacitance (resonant circuit).

The MCR tuning capacity was tested to the frequency range between $20 \mathrm{kHz}$ and $60 \mathrm{kHz}$. The tuning points when the MCR control DC current ranges from $0.0 \mathrm{~A}$ to $3.0 \mathrm{~A}$ is shown in Fig. 7. The main advantage of using the MCR is that the inductance $L$ can be continuously varied. The frequency adjustment range can varying 
continuously in between the two points of the maximum impedance as indicated in the Fig. 7.

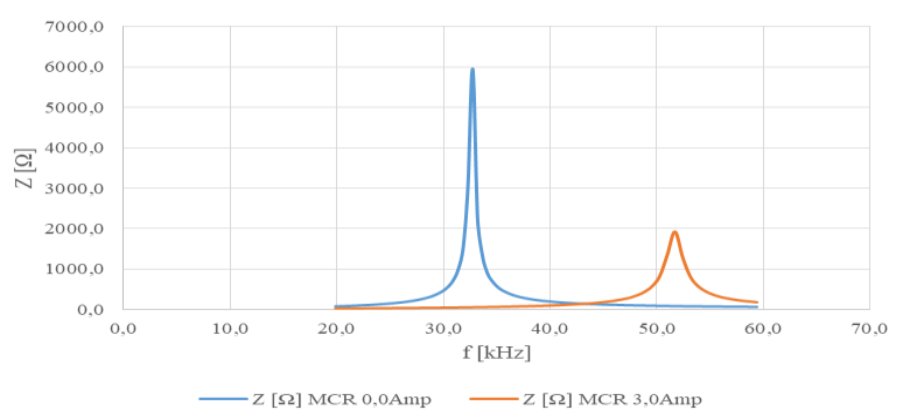

Fig. 7. Tuning points by varying the MCR DC control current for planar coil.

The magnetic field created by this coil (alone) was simulated when it is driven by a current of $15 \mathrm{~A}$ with the frequency of $17 \mathrm{kHz}$. The simulation results for planar coil: magnetic field distribution created for the frequency of $17 \mathrm{kHz}$ is shown in Fig. 8.

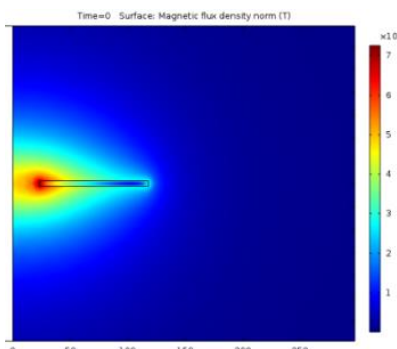

Fig. 8. Planar coil: magnetic field created for the frequency of $17 \mathrm{kHz}$.

\section{Conclusions}

The magnetic field created by the circular coil is more intense than that created by the planar coil. However, the spreading of the magnetic field created by the planar coil is larger by comparison of Fig. 5 and Fig. 8. This implies a greater efficiency in power transmission. Also note that although both coils have the same number of turns, the circular coil wire length was significantly higher yield which enables a greater magnetic field. The values of the impedances at resonance are larger in planar coil compared with the values of the circular coil, although obtained at different frequencies but within the close frequency range, few $\mathrm{kHz}$. Also, the frequency tuning range is greater in the planar coil is compared with that obtained in the circular coil, Fig. 4 and Fig. 7. 


\section{References}

1. Moradewicz, A.J, Kazmmierkowski, M.P.: High efficiency contactless energy transfer system with power electronic resonant converter. Bulletin of the Polish Academy of sciences, Technical Sciences 57(4), 375-381 (2009)

2. Qiu, C., Chau, K.Y., Liu, C., Chan, C.C.: Overview of wireless power transfer for electric vheicle charging. In: ESV27 International Battery, Hybrid and Fuel Cell Electric Vehicle Symposium, pp. 1-9, Barcelona, Spain (2013)

3. Umenei, A.E.: Understanding Low Frequency non-radiative Power Transfer. Fulton Innovation, LLC (2011)

4. Kurs, A., Karalis, A., Moffat, R., Joannopoulos, J.D., Fisher, P., Soljacic, M.: Wireless power transfer via strongly coupled magnetic resonances. Science 317(5834), 83-86 (2007)

5. Karalis, A., Joannopoulos, J.D., Soljacic, M.: Efficient wireless non-radiative mid-range energy transfer. Annals of Physic 323(1), 34-38 (2008)

6. Changsheng L., He, Z., Xiaohua, J.: Paramaters optimization for magnetic resonance coupling wireless power transmission. The Scientific World Journal 2014, 1-8 (2014)

7. Xiao, L., Ping, W., Dusit, N., Dong, I.K., Zhu, H.: Wireless charging technologies: fundamentals, standards and network applications. IEEE Communications Surveys and Tutorials 18(2), 1413-1452 (2016)

8. Hui, S.Y.R., Wenxing, Z., Lee, C.K.: A critical review of recent progress in mid-range wireless power transfer. IEEE Transactions on Power Electronics 29(9), 4500-4511 (2014)

9. Laugé, A., Hernantes, J., Sarriegi, J.M.: The role of critical infrastructures independences on the impacts caused by natural desasters. In: Critical Information Infrastructures Security, pp. 50-61, Springer (2013)

10 Steven, M.R., Peerenboom, J.P., Terrence, K.K.: Identifying, understanding and analyzing critical infrastructures independencies. IEEE Control Systems 21(6), 11-25 (2001)

11 Gomes, I.L.R., Pousinho, H.M.I., Melicio, R., Mendes, V.M.F.: Bidding and optimization strategies for wind-pv systems in electricity markets assisted by cps. Energy Procedia 106, 111-121 (2016)

12 Wang, J., Gharavi, H.: Power grid resilience. Proc. of the IEEE 105(7), 1199-1201 (2017)

13 Garcia-Villalobos, J., Zamora, I., San Martin, J.I., Junquera, I., Eguia, P.: Delivering energy from PEV batteries; V2G, V2B and V2H approaches. In: Int. Conf. on Renewable Energy and Power Quality, pp. 1-6, La Coruña, Spain (2015)

14 Rosa, E.B.: Calculation of the self-inductance of a single layer coils. Bulletin of the Bureau of Standards 2(2), 161-187 (1906)

15 Grover, F.W.: A comparison of the formulas for the calculation of the inductance of coils and spiral wound with wire of large cross sections. Bureau of the Standards Journal of Research 3, 163-190 (1929)

16 Benjamin, H.W., Brody, J.M., Gunbok, L., Joshua, R.S.: Optimal coil size ratios for wireless power transfer applications. In: IEEE International Symposium on Circuits and Systems, pp. 2045-2048, Melbourne, Australia (2014)

17 Baikova, E.N., Romba, L., Valtchev, S.S., Melicio, R., Pires, V.F., Krusteva, A., Gigov, G.: Electromagnetic field generated by a wireless energy transfer system: comparison of simulation to measurement, Journal of Electromagnetic Waves and Applications, 1-18, DOI: 10.1080/09205071.2017.1399832 (2017)

18 Romba, L.F., Valtchev, S.S., Melicio, R.: Improving magnetic coupling for battery charging through 3D magnetic flux. In: IEEE 17th International Conference on Power Electronics and Motion Control, pp. 291-297, Varna, Bulgaria (2016) 